hep-ph/9907498

PSU/TH/210

DESY 99-092

\title{
Transversity Distribution Does Not Contribute to Hard Exclusive Electroproduction of Mesons
}

\author{
J.C. Collins \\ Physics Department, Penn State University, 104 Davey Laboratory, University Park PA 16802, U.S.A. \\ M. Diehl \\ Deutsches Elektronen-Synchrotron DESY, 22603 Hamburg, Germany
}

(26 July 1999)

\begin{abstract}
We show that in hard exclusive electroproduction, ep $\rightarrow e V p$, the leading-twist hard-scattering coefficient for the production of a transversely polarized vector meson $V$ vanishes to all orders of perturbation theory. This implies that this process cannot be used to measure the skewed transversity distribution of quarks in a hadron. In contrast, a recent calculation obtained a non-zero value at NLO. We show that this calculation is incorrect because it failed to include the necessary collinear subtractions. Our method of proof also applies to other processes whose hard-scattering coefficients are constrained by chirality and helicity conservation, and thus validates helicity selection rules based on these symmetries.
\end{abstract}

12.38.Bx, 12.38.Qk, 13.60.Fz, 13.60.Le

\section{INTRODUCTION}

There has been much interest recently in hard exclusive electroproduction of vector mesons, $\gamma^{*} p \rightarrow V p$, at large photon virtuality and small invariant momentum transfer to the proton. In their paper [1] proving factorization for this process, Collins, Frankfurt and Strikman observed that the process appears to provide a new probe of the (skewed) transversity distribution of quarks in a proton, since this distribution appears in the factorization formula for production of transversely polarized vector mesons by longitudinally polarized virtual photons. Unfortunately, the lowest order coefficient function for this process vanishes, as found by Mankiewicz, Piller and Weigl [2]. Later Diehl, Gousset and Pire [3] showed how to extend this result to all orders in $\alpha_{s}$ by the use of the chiral symmetry of massless QCD and of rotational invariance.

But chiral invariance is broken by the axial anomaly of QCD, so Hoodbhoy and Lu [4] proposed that this would permit a non-zero coefficient function in non-leading order. Moreover, they found that in dimensional regularization the one-loop graphs for the process do indeed violate chirality conservation, and so they appear to obtain a non-zero coefficient function. They derived their result in $4-\epsilon$ dimensions from multiplying $1 / \epsilon$ factors due to divergences in the loop integrals by a factor of $\epsilon$ for the violation of chiral symmetry in the lowest order coefficient function. If their calculation is correct, it shows that we have a new process for measuring transversity distributions. This result would be important because of the paucity of leading twist processes that are sensitive to transversity distributions.

We show in this paper that their conclusion is, in fact, mistaken, and that the coefficient function does indeed vanish. The problem with the calculation of Ref. [4] is that to compute a one-loop contribution to a coefficient function from the corresponding graphs, one must subtract the zero-loop coefficient function times one-loop contributions to the parton distributions and distribution amplitudes. The subtractions exactly cancel the chirality violating term and result in a coefficient function that is zero. We explain this in detail in Sec. II.

Then, in Sec. III we generalize this result to all orders of perturbation theory. We complete the proof in Ref. [3] that the coefficient function vanishes. The vanishing is a consequence of properties of QCD that are related to chiral symmetry and remain true in the dimensionally regulated theory. In Sec. IV we comment on the relation between our result and the existence of the axial anomaly.

There are several other situations in perturbative QCD where chiral invariance is used to restrict the polarization states that occur at leading-twist. One example is hadronic helicity conservation [5], which holds in a number of exclusive processes. It applies for instance to the electromagnetic form factors of $\rho$-mesons, whose hardscattering coefficient is closely related to the one of exclusive meson production [3]. The objection of Hoodbhoy and $\mathrm{Lu}$ applies to these situations; the proofs previously given in the literature appear to be invalidated by the axial anomaly. The methods given in the present paper correct these proofs and show that the results obtained by chiral symmetry applied to hard-scattering coefficients are indeed valid.

Similarly, it is well known, on the basis of chirality 


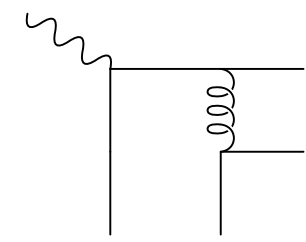

FIG. 1. A lowest order graph for the hard-scattering coefficient.

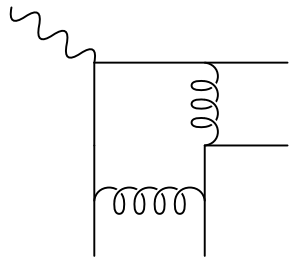

FIG. 2. An NLO graph for the hard-scattering coefficient.

conservation arguments, that chiral odd parton densities and fragmentation functions occur in pairs but not singly [6]. Again the methods presented in the present paper ensure that these results are valid despite the anomalous violation of chiral symmetry.

\section{ONE-LOOP CALCULATION WITH SUBTRACTIONS}

If the calculation of the coefficient function for our process could be performed in massless QCD without a regulator, then one could immediately apply the proof of Ref. 3] to show that the coefficient function vanishes. But there are collinear divergences, so a regulator must be applied. When dimensional regularization with $4-\epsilon$ dimensions is used, it is easy [4] to see that tree graphs, like Fig. 1, for the coefficient function for our process are proportional to $\epsilon$. When the graph is contracted with chiral odd quark distribution amplitudes, one obtains a factor of the form

$$
\gamma^{\lambda} \gamma^{+} \gamma^{i} \gamma_{\lambda}
$$

where $\lambda$ is summed over and $i$ is a transverse index. Here and in the following we use light-cone notation, $\gamma^{ \pm}=$ $\left(\gamma^{0} \pm \gamma^{3}\right) / \sqrt{2}$. The matrix in (1) equals $-\epsilon \gamma^{+} \gamma^{i}$, and hence in 4 dimensions the coefficient function is zero.

Consider now a one-loop graph, such as Fig. 2. It has collinear divergences when one or other of the gluons becomes parallel to the attached quark lines. The resulting $1 / \epsilon$ multiplies a factor $\epsilon$ of the same origin as in a tree graph and gives a nonzero result at $\epsilon=0$. Hoodbhoy and $\mathrm{Lu}$ [4] computed the sum over all graphs and found that the result remains non-zero.

However, this does not represent the full calculation of the coefficient function. Schematically the factorization theorem is

$$
A=H * f * \phi,
$$

where the star denotes convolution, $A$ is the amplitude for the process, up to power corrections in the photon virtuality, $H$ is the hard-scattering function, $f$ is the skewed parton distribution in the target, and $\phi$ is the meson distribution amplitude. Let us apply the theorem to massless quark states and take the one-loop terms (of order $\left.\alpha_{s}^{2}\right)$. We have

$$
A_{1}=H_{1} * f_{0} * \phi_{0}+H_{0} * f_{1} * \phi_{0}+H_{0} * f_{0} * \phi_{1},
$$

where the subscripts represent the number of loops in perturbation theory. At one-loop order, the value of $H$ is thus calculated by

$$
H_{1}=H_{1} * f_{0} * \phi_{0}=A_{1}-H_{0} * f_{1} * \phi_{0}-H_{0} * f_{0} * \phi_{1},
$$

the lowest order values $f_{0}$ and $\phi_{0}$ on quark states being trivial delta functions.

Now, the factor of $1 / \epsilon$ that gave the nonzero result for $A_{1}$ is obtained from the very collinear regions that are to be subtracted: The subtractions ensure that the hard-scattering coefficient $H_{1}$ is dominated by the region where all the lines have virtualities of order $Q^{2}$. The collinear divergence in the subtractions in Eq. (4) is multiplied by a factor proportional to $\epsilon$, namely the lowestorder coefficient $H_{0}$, and one obtains exactly the same factors of $1 / \epsilon$ and $\epsilon$ as in the corresponding contributions to $A_{1}$. Thus the sum of the subtraction terms cancels the finite nonzero term in $A_{1}$, so that $H_{1}$ vanishes at $\epsilon=0$. We will show this in more detail in Sec. III. When one takes parton distributions and distribution amplitudes that are chirally even instead of chirally odd then the corresponding $1 / \epsilon$ factors are not multiplied by a factor that vanishes at $\epsilon=0$, and in that case the subtraction terms cancel the divergent terms so that $H_{1}$ is finite. This cancellation is guaranteed by the factorization theorem.

A clear symptom that the result of Ref. [4] cannot provide a correct calculation of the coefficient function is that it comes from a collinear region. . Coefficient functions, however, are collinear safe quantities.

Often one gets the impression from the literature that obtaining a one-loop coefficient function is merely a matter of subtracting $1 / \epsilon$ poles from the unsubtracted graphs. This is not the case. Fundamentally, the correct subtractions have the form shown in Eq. (4); this is

\footnotetext{
${ }^{*}$ Hoodbhoy and Lu also obtain a finite term by multiplying the factor $\epsilon$ from the tree graphs with a $1 / \epsilon$ ultraviolet pole from the wave function renormalization factors of the external quark lines. However, if one works with renormalized amplitudes, as is always correct, the ultra-violet divergences are cancelled by renormalization counterterms. Moreover, no factors associated with propagator corrections on external lines need be considered when calculating a hard-scattering coefficient, since they identically cancel in the subtractions.
} 


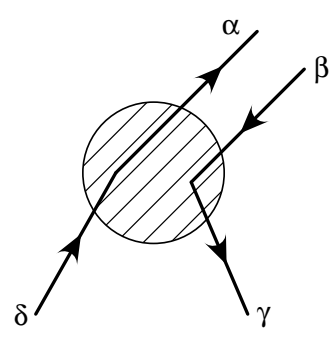

(a)

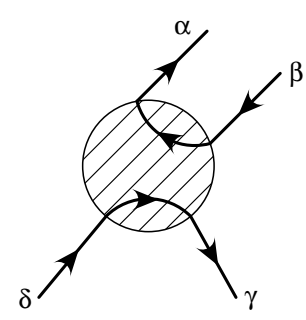

(b)
FIG. 3. Hard-scattering coefficient with spinor labels. The external photon is not shown, and diagrams (a) and (b) show the two possibilities for connecting the external quark lines.

necessary to avoid double counting [7]. Only if the Born graphs are independent of $\epsilon$ is the subtraction formula (何) equivalent to the subtraction of poles. The difference between the correct and incorrect procedures is, of course, particularly noticeable when the Born graphs are proportional to $\epsilon$ so that there is no pole at the one-loop level.

\section{GENERAL PROOF}

In this section we close a loophole in the proof given in [3] that the coefficient function for the electroproduction of transversely polarized vector mesons vanishes to all orders of perturbation theory. That proof does not treat the complications due to the fact that chiral symmetry is broken in massless QCD whenever the theory is regulated.

With the spin-dependent factors for the external quarks removed, the coefficient function has four Dirac indices: $H_{\alpha}{ }^{\beta} \gamma^{\delta}$, as illustrated in Fig. 3. The lower two quark lines are associated with the proton, and have momenta in the $+z$ direction. The upper two lines are associated with the meson and have momenta in the $-z$ direction. Since $H$ is a hard-scattering coefficient, all its external momenta are massless and on-shell, and all internal masses are set to zero.

The theorem we will prove is that $H$ vanishes when contracted with the Dirac matrices appropriate for a transversity distribution in the proton and for a transversely polarized meson:

$$
H_{\alpha}{ }^{\beta} \gamma^{\delta}\left(\gamma^{+} \gamma^{i}\right)_{\beta}^{\alpha}\left(\gamma^{-} \gamma^{j}\right)_{\delta}^{\gamma}=0 .
$$

Here $i$ and $j$ are transverse indices associated with the polarization states of the meson and proton.

There are two possibilities for the topological connection of the external quark lines. The first case is shown in Fig. 3(a), where the lines from the proton join to the lines from the meson. The second case is shown in Fig. $3(\mathrm{~b})$, where the lines from the proton join to each other and the lines from the meson join to each other. As we will discuss below, there is an odd number of Dirac matrices on each of the internal lines of the coefficient function. Since the trace of an odd number of Dirac matrices vanishes, graphs of type (b) give zero when contracted with the external Dirac matrices in Eq. (5). Hence only graphs of type (a) need to be considered.

\section{A. Previous proof}

Ref. [3] gave two proofs: One is that chirality is conserved in massless QCD; this forces the initial and final quark states in Fig. 3 to have $J_{z}$ differing by two units, which is prohibited by angular momentum conservation because the reaction is collinear and the photon can at most provide one unit of $J_{z}$. The second proof, which translates this into algebra, is to observe that the Feynman rules of massless QCD imply that $H_{\alpha}{ }^{\beta}{ }_{\gamma}{ }^{\delta}$ is the tensor product of two fermion lines, each containing an odd number of Dirac matrices, as in Fig. 1 or 2 . The only possibilities that have the correct transformation under rotations about the $z$-axis give Eq. (5).

The first proof is incomplete as it does not discuss possible problems caused by the anomalous breaking of chiral symmetry in massless QCD.

For the second proof we remark that the property of having an odd number of Dirac matrices along each fermion line remains true in the dimensionally regularized theory. This is not changed by manipulations such as decreasing the number of matrices by using $\left\{\gamma^{\mu}, \gamma^{\nu}\right\}=2 g^{\mu \nu}$ in an arbitrary number of space-time dimensions.

Now, the calculation of Ref. [4] tells us that Eq. (5) is false when applied to unsubtracted amplitudes. This can be understood by observing that in order to use chirality conservation and to perform the algebraic manipulations in the second proof of Ref. [3] one must be in 4 dimensions. Without subtractions, however, one cannot set $\epsilon=0$ in $H_{\alpha}{ }^{\beta}{ }^{\delta}$ because of the pole $1 / \epsilon$ from the momentum integration. Hoodbhoy and Lu obtain a finite result only after multiplying with the Dirac matrices for the external lines, which provide a factor of $\epsilon$. In this way chirality conservation is broken because of non-trivial contributions from the Dirac matrices in the unphysical dimensions. If

\section{B. Completed proof}

We now show that in the hard-scattering coefficient $H_{\alpha}{ }^{\beta} \gamma^{\delta}$, once one has made the appropriate subtractions and taken the limit $\epsilon=0$, chirality is conserved to all orders in perturbation theory, so that both proofs of Ref. [3] apply and Eq. (5) is correct. We start from the Feynman diagrams for the hard scattering, without multiplying by

\footnotetext{
${ }^{\dagger}$ Remember that for $\mu \neq 0,1,2,3$ the matrices $\gamma^{\mu}$ do not anticommute with $\gamma_{5}=i \gamma^{0} \gamma^{1} \gamma^{2} \gamma^{3}$.
} 
the Dirac factors of the external quark lines, and proceed in several steps.

1. Regulate the loop integrals by going to $4-\epsilon$ dimensions.

2. Perform the usual ultraviolet subtractions in the diagrams, as specified by the counterterms in the Lagrangian. Soft or infra-red divergences cancel after summing over all graphs at a given order in $\alpha_{s}$ [1], so that after this step only collinear divergences are left.

3. Perform the subtractions for the collinear regions, as in Eq. (4) and its generalizations to higher order. Note that the subtraction scheme is defined by the ultra-violet renormalization that is used to define the parton densities. As in step 2, one has a choice of subtraction scheme and may for instance use the $\overline{\mathrm{MS}}$ prescription.

4. Remove the regulator, i.e., take the limit $\epsilon=0$. The crucial fact is that one obtains a finite result for $H_{\alpha}{ }^{\beta}{ }_{\gamma}{ }^{\delta}$ before contracting with any Dirac matrices. This follows from the general construction of the factorization proof [1]: Momentum regions for Feynman graphs for the overall process are characterized by a division into subgraphs associated with the three building blocks $H, f$ and $\phi$ of the factorization formula (2). To show that the hardscattering coefficient $H$ is finite and dominated by large virtualities does not require use of the Dirac algebra. Hence we can set $\epsilon=0$ before projecting out any particular Dirac structure.

None of these steps impinges on the fact that for each quark line we have a string of an odd number of Dirac matrices. This is ensured by the vector coupling structure of the Lagrangian and of the counterterms. Now, a product of an odd number of basic Dirac matrices in 4 dimensions conserves the chirality of the external lines, from which follows the same property for $H$. This completes the proof.

Let us illustrate these steps by the following integral that contains a typical structure in the one-loop contribution to $H_{\alpha}{ }^{\beta} \gamma^{\delta}$. Without subtractions it is

$$
I_{\alpha}{ }^{\beta} \gamma^{\delta}=\left(\gamma^{\lambda}\right)_{\gamma}{ }^{\beta}\left(\gamma_{\lambda}\right)_{\alpha}{ }^{\delta} \mu^{\epsilon} \int \frac{d^{2-\epsilon} k_{T}}{(2 \pi)^{2-\epsilon}} \frac{f\left(k_{T}\right)}{k_{T}^{2}} .
$$

This contains an integral over transverse momentum which becomes divergent as $k_{T} \rightarrow 0$ when $\epsilon=0$. The factor $f\left(k_{T}\right)$ represents the rest of the integrand and provides a cut-off at large $k_{T}$, so that there are no ultraviolet divergences in this case. The contribution appropriate for the coefficient of a transversely polarized meson is obtained by contracting with $\left(\gamma^{+} \gamma^{i}\right){ }_{\beta}{ }^{\alpha}$; then the Dirac algebra gives a factor proportional to $\epsilon$.

After subtraction of the collinear divergence we obtain

$$
\begin{aligned}
\bar{I}_{\alpha}{ }^{\beta} \gamma^{\delta}=\left(\gamma^{\lambda}\right)_{\gamma}{ }^{\beta}\left(\gamma_{\lambda}\right)_{\alpha}{ }^{\delta} & \left\{\mu^{\epsilon} \int \frac{d^{2-\epsilon} k_{T}}{(2 \pi)^{2-\epsilon}}\left[\frac{f\left(k_{T}\right)}{k_{T}^{2}}-\frac{f(0)}{k_{T}^{2}}\right]\right. \\
& \left.+\frac{f(0)}{4 \pi}\left(\frac{2}{\epsilon}+\ln 4 \pi-\gamma\right)\right\} .
\end{aligned}
$$

Here the second term inside the square brackets represents an integrand for the parton density or the meson distribution amplitude. It cancels the singularity in the integrand but introduces an ultra-violet divergence that we renormalize by the last term, which is an $\overline{\mathrm{MS}}$ counterterm. The factor that multiplies the Dirac matrices, i.e., the factor in braces, is finite as $\epsilon \rightarrow 0$.

A convenient way [8] of representing the counterterm is as an integral over $k_{T}$, so that at $\epsilon=0$ we have a finite integral for $\bar{I}$ :

$$
\begin{aligned}
& \bar{I}_{\alpha}^{\beta}{ }^{\delta}=\left(\gamma^{\lambda}\right)_{\gamma}^{\beta}\left(\gamma_{\lambda}\right)_{\alpha}^{\delta} \\
& \int_{0}^{\infty} \frac{d k_{T}^{2}}{4 \pi}\left[\frac{f\left(k_{T}\right)}{k_{T}^{2}}-\frac{f(0)}{k_{T}^{2}} \theta\left(\mu-k_{T}\right)\right] .
\end{aligned}
$$

Clearly, we now have 4-dimensional Dirac matrices multiplying a finite integral. This is the final result for the contribution to $H$, after step 4 of the construction, and chirality conservation clearly holds.

A few remarks are in order about multiplying with the Dirac matrices for the external lines. Clearly this multiplication must not be done before regularization, otherwise one risks multiplying an infinity from the loop integrals by a zero from the Dirac algebra, and thereby losing control over the calculation. It can be done after step 1 as all expressions are well defined, and if one correctly performs all subtractions, one will obtain the correct result. However, as explained at the end of Sec. III A, it is difficult to keep track of chirality in $4-\epsilon$ dimensions, where this symmetry is broken. Postponing the multiplication by external factors until after step 4 has been performed enables us to show that no chirality breaking effects from the extra dimensions survive in the hardscattering coefficient.

Dimensional regularization is not the only possibility to regulate the collinear divergences in the hard scattering. For an alternative proof one could let the external quark lines in $H$ be slightly off-shell. (One may want to do this without giving them transverse momentum so as to keep rotation invariance in the $x-y$ plane, which is the other essential ingredient in the proof next to chirality conservation.) Then one can take the limit $\epsilon=0$ already after step 2. Removing the regulator in step 4 then means that we put the external lines back on shell.

Clearly our arguments leading to chirality conservation in the hard-scattering coefficient are not specific to exclusive vector meson production, and generalize to other processes.

\section{THE AXIAL ANOMALY}

Let us make some remarks about how one can understand our result in the light of the axial anomaly in QCD. 
We have shown that chirality is conserved for the coefficient function $H_{\alpha}{ }^{\beta}{ }^{\delta}$, i.e., that it is invariant under a chiral transformation of the external quark legs:

$$
H_{\alpha}{ }^{\beta} \gamma^{\delta}=U_{\alpha}{ }^{\alpha^{\prime}} U_{\gamma}{ }^{\gamma^{\prime}} H_{\alpha^{\prime}}{ }^{\beta^{\prime}}{ }_{\gamma^{\prime}}^{\delta^{\prime}} U_{\beta^{\prime}}{ }^{\beta} U_{\delta^{\prime}}{ }^{\delta}
$$

where $U=\exp \left(i \omega \gamma_{5}\right)$ with a real parameter $\omega$. The invariance follows simply from the fact that $H$ is a tensor product of strings of an odd number of basic 4dimensional Dirac matrices (i.e., of $\gamma^{0}, \ldots, \gamma^{3}$ ).

To obtain this result we used arguments about the counting of Dirac matrices that remain valid in the dimensionally regulated theory. However, we only invoke chirality once we are back in 4 dimensions. We have not made use of the Noether theorem and the axial current or axial charge. To prove the Ward identity associated with chiral symmetry requires more than we needed in our proof, and, as is well known, the proof that the axial current is conserved fails.

Even though the axial anomaly in QCD can be calculated in one-loop perturbation theory, its existence does not of itself imply broken chiral symmetry. The anomaly is merely an enabling result that permits anomalous chiral symmetry breaking. It tells us that the divergence of the axial current, $\partial \cdot j_{5}$, is another operator, proportional to $F \cdot \tilde{F}$, but it does not tell us that matrix elements of this operator are non-zero. Note that by its definition $F \cdot \tilde{F}$ is a pure divergence, although of a non-gauge invariant operator. The anomaly is a necessary but not sufficient condition for the breaking of chiral symmetry. As has been observed in other work [9], the actual breaking of chiral symmetry in QCD is purely non-perturbative. There is no anomalous breaking of chiral symmetry in any finite order of perturbation theory, provided that a genuinely infrared safe quantity is calculated: hard-scattering coefficients respect chiral invariance in the sense of Eq. (9). Notice that in the context of the spin crisis the axial anomaly appears in the hadronic matrix elements that define the parton densities, i.e., in non-perturbative quantities.

\section{CONCLUSIONS}

We have shown explicitly that chiral symmetry for massless external fermion lines is preserved order by order in perturbative QCD, provided that an infrared safe quantity is calculated. This implies that, in the electroproduction of mesons, the hard-scattering coefficient vanishes when one takes the component that couples to a transversity distribution. Thus, the proposal of Collins, Frankfurt and Strikman [1] to use this process for measuring transversity distributions does not work. The claim of Hoodbhoy and Lu [4] to the opposite effect fails because the authors omitted to make the collinear subtractions necessary in a correct calculation of a hard-scattering coefficient. Our proof completes that given by Diehl, Gousset and Pire [3].
The methods we employ apply to other cases, and show that the application of chiral symmetry to hardscattering coefficients is generally valid.

Our result is in one sense negative, since it shows that electroproduction of mesons cannot be used to measure transversity distributions. However it also provides a helicity selection rule: If any exclusive electroproduction of transversely polarized vector mesons is observed, it must be due to a power correction. In this respect the process is very similar to exclusive processes where hadron helicity conservation holds, such as electron-hadron or hadron-hadron scattering at large momentum transfer, or to the structure function $g_{2}$, which provides a probe of higher-twist physics uncontaminated by leading twist.

\section{ACKNOWLEDGMENTS}

This work was supported in part by the U.S. Department of Energy under grant number DE-FG02-90ER40577 , and in part by the TMR program of the European Union under contract number FMRX-CT98-0194. M.D. would like to thank M. Beneke, T. Gousset, B. Pire and A.V. Radyushkin for discussions and E. Pilon for correspondence. J.C. would like to thank M. Eides for discussions.

[1] J.C. Collins, L. Frankfurt and M. Strikman, Phys. Rev. D56, 2982 (1997).

[2] L. Mankiewicz, G. Piller and T. Weigl, Eur. Phys. J. C5, 119 (1998).

[3] M. Diehl, Th. Gousset and B. Pire, Phys. Rev. D59, 034023 (1999).

[4] P. Hoodbhoy and W. Lu, "Electroproduction of Transversely Polarized Vector Mesons Via A Quantum Mechanical Anomaly," hep-ph/9902286.

[5] S.J. Brodsky and G.P. Lepage, Phys. Rev. D24, 2848 (1981).

[6] X. Artru and M. Mekhfi, Z. Phys. C45, 669 (1990); J.L. Cortes, B. Pire and J.P. Ralston, Z. Phys. C55, 409 (1992);

J.C. Collins, Nucl. Phys. B396, 161 (1993).

[7] J.C. Collins, D.E. Soper and G. Sterman, "Factorization of Hard Processes in QCD", in: Perturbative Quantum Chromodynamics, ed. A.H. Mueller, (World Scientific, Singapore, 1989); and references therein.

[8] J.C. Collins, J. Phys. G 17, 1547 (1991).

[9] E. Witten, Nucl. Phys. B156, 269 (1979);

D.I. D'yakonov and M.I. Eides, Zh. Eksp. Teor. Fiz. 81, 434 (1981), Engl. transl.: Sov. Phys. JETP 54, 232 (1981); and references therein. 